\title{
Performance and physics measurements at protoDUNE-SP
}

\author{
Stefania Bordoni*, for the DUNE Collaboration \\ CERN \\ E-mail: stefania.bordoni@cern.ch
}

The protoDUNE Single Phase (protoDUNE-SP) TPC is a prototype for the DUNE far detector and it is currently under construction at CERN. It will not only serve as a test-bed for the engineering design and construction techniques but will also provide a set of key measurements for the future DUNE far detector.

The new extension of the $\mathrm{H} 4$ beam-line at CERN will provide electron, muon and hadron beams with energies in the range of sub-GeV to a few $\mathrm{GeV}$. The data collected during the test beams will be analysed to assess the performance of a state-of-the-art LAr TPC. For such detectors the event reconstruction is challenging and test beam data will allow the performance of the different reconstruction algorithms, which also involve machine-learning techniques, to be benchmarked and improved. Furthermore, important physics measurements are foreseen with protoDUNE-SP. For example, the study of pion interactions in argon using test beams is fundamental to understand some of the processes that will be seen as part of the primary neutrino interactions and which would modify the event signature in the DUNE detectors. ProtoDUNE-SP will provide new measurements of the pion-Ar cross-section, for both positive and negative charges, which will make a vital contribution to improve the current, very limited knowledge of such interactions.

The European Physical Society Conference on High Energy Physics

5-12 July, 2017

Venice

${ }^{*}$ Speaker. 


\section{Introduction}

The DUNE (Deep Underground Neutrino Experiment) is one of the new generation of LongBaseline Neutrino experiments foreseen to push the frontiers of neutrino physics and proton decay for the next two decades [1]. To validate the technology and the design of a module of the DUNE far detector (FD), a large engineering and physics prototype for a single phase liquid argon (LAr) Time Projection Chamber (TPC) is being constructed at CERN.

\section{A big LAr TPC in a test beam at CERN}

The large single-phase (SP) LAr TPC prototype, called protoDUNE-SP, is contained in a $10 \mathrm{~m}$ edge cubic cryostat (outer dimensions) with a capacity of about 800 tonnes of LAr. The detector has been designed to use the technical solutions and full-size individual elements foreseen for the DUNE FD detector modules. Even at 1:20 of the final size of a DUNE FD module, protoDUNE is the largest LAr TPC ever built. With its cathode located at the center of the volume with a nominal bias voltage of $-180 \mathrm{kV}$, a uniform electric field of $500 \mathrm{~V} / \mathrm{cm}$ will drift electrons laterally toward the anode planes for a maximum distance of $3.6 \mathrm{~m}$. The readout of the ionised and scintillation signals from the LAr is done by six modules of Anode Plane Assembly (APA) of $6 \mathrm{~m}$ x $2.3 \mathrm{~m}$ each which are composed by three planes of wires spaced at $5 \mathrm{~mm}$ and 10 wavelength-shifting bars read by silicon photomultipliers (SiPM). Figure 1 show an illustration of the protoDUNE-SP TPC with its major components. A new extension of the H4 SPS beam has been conceived to expose the detector to charged particles $\left(\mathrm{e}^{ \pm}, \mu^{ \pm}, \mathrm{p}, \pi^{ \pm}, \mathrm{K}^{ \pm}\right.$) of energies between 0.5 and $6 \mathrm{GeV}$. This new beam line will be instrumented with beam monitors, Time of Flight detectors and Cherenkov counters to identify the particles before entering the detector and measure their momenta with a spread of 5$7 \%$. The design of the detector has been driven by the physics performance requirements, enabling for high efficiency reconstruction and identification of the particles inside the entire active volume of the LAr TPC. Argon purity, drift distance, wire pitch are for example some of the parameters which determine the capability of the detector to identify Minimum Ionising Particles. A detailed list of physics and detector requirements are shown in Tables 1 (a) and (b).

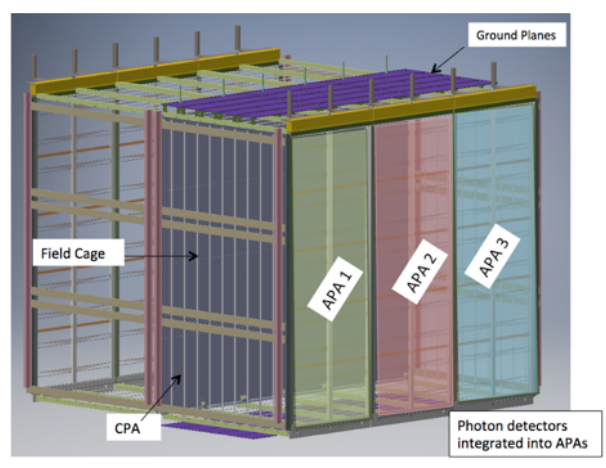

Figure 1: Illustration of the protoDUNE-SP LAr TPC and its principal components. 


\begin{tabular}{lr}
\hline Requirement & Value \\
\hline \hline MIP Identification & $100 \%$ efficiency \\
High efficiency for charge reconstruction & $>90 \%$ for $>100 \mathrm{MeV}$ \\
Vertex Resolution (x,y,z) & $(1.5 \mathrm{~cm}, 1.5 \mathrm{~cm}, 1.5 \mathrm{~cm})$ \\
Particle Identification & $<18 \%$ for non-contained \\
Muon Momentum Resolution & $<5 \%$ for contained \\
& $<1^{\circ}$ \\
Muon Angular Resolution & $1-5 \%$ \\
Stopping Hadrons Energy Resolution & $<10^{\circ}$ \\
Hadron Angular Resolution & $>90 \%$ \\
Shower identification & $<1 \%$ \\
Electron efficiency & $<1^{\circ}$ \\
Photon mis-identification & $<5 \%$ \\
Electron Angular Resolution & \\
Electron Energy Scale Uncertainty & \\
\hline
\end{tabular}

\begin{tabular}{ll}
\hline Requirement & Value \\
\hline \hline Efield & $550 \mathrm{~V} / \mathrm{cm}$ \\
Electron drift time & $2.25 \mathrm{~ms}$ \\
Purity & $<100 \mathrm{ppt} \mathrm{O}_{2}$ equivalent \\
Signal to Noise ratio & $>9: 1$ \\
Spatial resolution & $<1.5 \mathrm{~cm}$ (all directions) \\
\hline
\end{tabular}

(b)

(a)

Table 1: Some of the physics (a) and the detector (b) requirements for protoDUNE-SP [2].

\section{Event reconstruction in protoDUNE-SP}

Liquid argon TPCs provide very high resolution 3D events. However the interpretation of LAr data and the reconstruction of the events is very challenging because the large amount of information per event, the high rate of particle interactions and scattering and the projection of the $3 \mathrm{D}$ event into three 2D spaces (readout of the APA wires as a function of the time). The event reconstruction [2] deals with several steps which goes from the reduction of the electronic noise, hit finding (e.g. signals above a given threshold) and their attribution first to a DAQ channel and later to a wire segment. Several algorithms are available to group the hits into tracks or clusters objects in each view (1 view per APA) separately and then 3D hypotheses are built by comparing the $2 \mathrm{D}$ images in separate planes. The algorithms currently in use to reconstruct the events are based on a combination of traditional tracking algorithms and new multivariate techniques. As shown in Figure 2, performance studies based on simulation show excellent muon tracking efficiencies as well as 3D vertex resolution of the order of $1 \mathrm{~cm}$ fulfilling the nominal requirements. A new approach based on the use of Convolutional Neural Networks is being developed to help to disentangle between track-like and shower-like objects which is a crucial requirement to ensure a better estimation of the electromagnetic part of the event.

\section{Physics measurements}

Using the dedicated very low energy extension of the H4 beam line, protoDUNE-SP will also perform some physics measurements of interest for the neutrino community and in particular for the experiments based on LAr. Neutrino interactions can produce low energy pions and protons whose detection is fundamental to either disentangle between interaction type or study the re-interaction of the particles when living the nucleus (e.g. Final State Interaction or Secondary Interaction). If those processes occur, the signature of the event can dramatically change with deep impact on the inferred neutrino energy. Such measurements are very limited or even missing depending on 


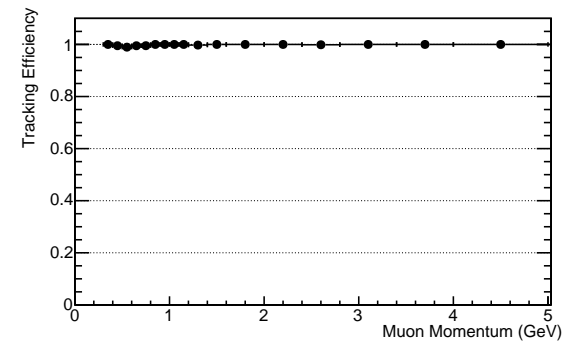

(a)

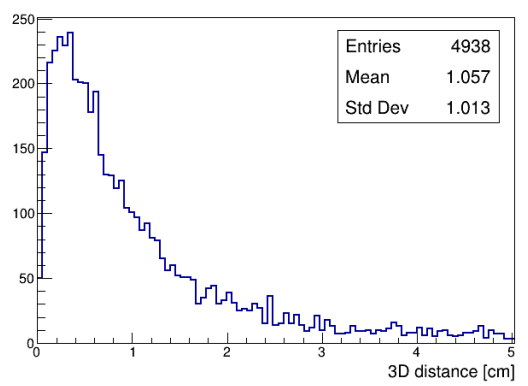

(b)

Figure 2: Muon tracking efficiency (a) and vertex resolution at the interaction point for pions (b).

the neutrino target nuclei but they are critical to keep the systematic uncertainties affecting the oscillation analyses under control.

ProtoDUNE-SP is preparing analyses to measure the positive and negative pion cross section in Argon in a range of energies between 1 and $6 \mathrm{GeV}$. The current state-of-the art measurements are limited to extrapolation at very low energies (order of few hundreds of $\mathrm{MeV}$ ) from other nuclei [3] and to the measurement recently performed by LArIAT [4]. The proton-Ar cross section is also of interest and is considered for physics measurement with protoDUNE beam data.
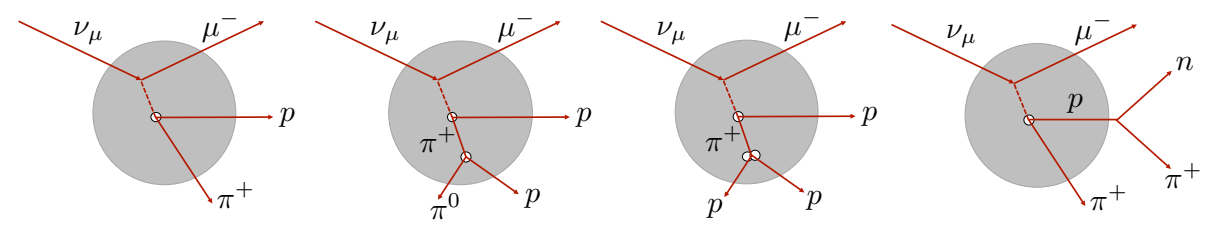

Figure 3: Example of processes which can modify the signature of the event.

\section{Conclusion}

ProtoDUNE-SP is an 800 tonnes LAr TPC being constructed at CERN which will serve as engineering and physics prototype for the DUNE far detector modules. Large efforts are currently on-going in the preparation of data treatments which are advancing the state-of-the art in LAr event reconstruction algorithms and software suites. Finally, physics measurements of charged particle cross sections in Argon at energies relevant to DUNE neutrino interactions are foreseen already with the first beam data from the new extension of the CERN H4 beam line.

\section{References}

[1] The DUNE Collaboration, CDR Volume 2, arXiv1512.06148.

[2] The DUNE collaboration, protoDUNE-SP TDR, arXiv:1706.07081.

[3] D. Ashery et al., True absorption and scattering of pions on nuclei, Phys. Rev. C 23, 2173 (1981).

[4] P. Hamilton (the LArIAT Collaboration), arXiv1611.00821. 